Received: 18.06 .2018

Revised: 07.09.2018

Accepted: 19.10 .2018

DOI: $10.17804 / 2410-9908.2018 .6 .059-082$

\title{
INFLUENCE OF DIFFUSION MODELS ON CHEMICAL REACTION FRONT KINETICS
}

\author{
P. M. Grigoreva ${ }^{\text {a)* }}$ and E. N. Vilchevskaya ${ }^{\text {b) }}$ \\ Institute for Problems in Mechanical Engineering, RAS, \\ 61 Bolshoy Ave., V.O., St. Petersburg, 199178, Russian Federation \\ a) (D) https://orcid.org/0000-0001-9846-7888 polina.grigoreva239@ @mail.com; \\ *Corresponding author. E-mail: polina.grigoreva239@gmail.com \\ Address for correspondence: V.O., Bolshoy pr., 61, 199178, St. Petersburg, Russian Federation \\ Tel.: +7 (812) 32147 78; fax: +7 (812) 3214771
}

\begin{abstract}
A linear-elastic body is considered, in which a chemical reaction localized at the front is maintained, supported by the diffusion of the gaseous component through the layer of the newlyformed material. The comparative influence of mechanical stresses on the kinetics of the chemical reaction front is studied by taking into account the contribution of stresses to the surface reaction rate through the chemical affinity tensor and to the diffusion process through various stress dependences of the diffusion coefficient. As an example, the propagation of the centrally symmetric and axisymmetric reaction fronts in various boundary-value problems is considered with the use of different diffusion models.
\end{abstract}

Keywords: mechanochemistry, tensodiffusion, non-equilibrium thermodynamics.

\section{Acknowledgment}

The research was supported by the Russian Scientific Foundation, project No. 18-19-00160.

\section{References}

1. Kao Dah-Bin, McVittie J.P., Nix W.D., Saraswat K.C. Two-dimensional silicon oxidation experiments and theory. In: 1985 International Electron Devices Meeting, IEEE, 1985, pp. 388-391. DOI: 10.1109/iedm.1985.190982.

2. Kao Dah-Bin, McVittie J.P., Nix W.D., Saraswat K.C. Two-dimensional thermal oxidation of silicon-I. Experiments. IEEE Transactions on Electron Devices, 1987, vol. 34, iss. 5, pp. 1008-1017. DOI: 10.1109/T-ED.1987.23037.

3. Sutardja P., Oldham W.G. Modeling of stress effects in silicon oxidation. IEEE Transactions on Electron Devices, 1989, vol. 36, no. 11, pp. 2415-2421. DOI: 10.1109/16.43661.

4. Paul Aloke, Laurila Tomi, Vuorinen Vesa, Divinski Sergiy V.I. Thermodynamics, diffusion and the Kirkendall effect in solids, Springer, 2014. DOI: 10.1007/978-3-319-07461-0.

5. Cui Z., Gao F., Qu J. Interface-reaction controlled diffusion in binary solids with applications to lithiation of silicon in lithium-ion batteries. Journal of the Mechanics and Physics of Solids, 2013, vol. 61, no. 2, pp. 293-310. DOI: 10.1016/j.jmps.2012.11.001.

6. Knyazeva A.G. Model of medium with diffusion and internal surfaces and some applied problems. Mater. Phys. Mech., 2004, vol. 7, no. 1, pp. 29-36.

7. Knyazeva A.G. Cross effects in solid media with diffusion. Journal of Applied Mechanics and Technical Physics, 2003, vol. 44, no. 3, pp. 373-384. DOI: 10.1023/A:1023485224031. 
8. Toribio J., Kharin V., Lorenzo M., Vergara D. Role of drawing-induced residual stresses and strains in the hydrogen embrittlement susceptibility of prestressing steels. Corrosion Science, 2011, vol. 53, no. 10, pp. 3346-3355. DOI: 10.1016/j.corsci.2011.06.012.

9. Cui Z., Gao F., Qu J. A finite deformation stress-dependent chemical potential and its applications to lithium ion batteries. Journal of the Mechanics and Physics of Solids, 2012, vol. 60, no. 7, pp. 1280-1295. DOI: 10.1016/j.jmps.2012.03.008.

10. Freidin A.B. Chemical affinity tensor and stress-assist chemical reactions front propagation in solids. In: ASME 2013 International Mechanical Engineering Congress and Exposition, American Society of Mechanical Engineers, 2013, pp. V009T10A102-V009T10A102.

11. Freidin A.B. On the chemical affinity tensor for chemical reactions in deformable materials. Mechanics of Solids, 2015, vol. 50, no. 3, pp. 260-285. DOI: 10.3103/S0025654415030048.

12. Freidin Alexander B., Korolev Igor K., Aleshchenko Sergey P., Vilchevskaya Elena N. Chemical affinity tensor and chemical reaction front propagation: theory and FE-simulations. International Journal of Fracture, 2016, vol. 202, no. 2, pp. 245-259. DOI: 10.1007/s10704-016-0155-1.

13. Freidin A.B., Vilchevskaya E.N., Korolev I.K. Stress-assist chemical reactions front propagation in deformable solids. International Journal of Engineering Science, 2014, vol. 83, pp. 57-75. DOI: 10.1016/j.ijengsci.2014.03.008.

14. Freidin A., Morozov N., Petrenko S., Vilchevskaya E. Chemical reactions in spherically symmetric problems of mechanochemistry. Acta Mechanica, 2016, vol. 227, no. 1, pp. 43-56. DOI: $10.1007 / \mathrm{s} 00707-015-1423-2$.

15. Glansdorff P., Prigogine I. Thermodynamic theory of structure, stability and fluctuations. American Journal of Physics, 1973, vol. 41, no. 1, pp. 147-148.

16. Grinfel'd M.J., ed. Thermodynamic Methods in the Theory of Heterogeneus Systems, Longman Scientific and Technical, 1991.

17. Kao Dah-Bin, McVittie J.P., Nix W.D., Saraswat K.C. Two-dimensional thermal oxidation of silicon. II. Modeling stress effects in wet oxides. IEEE transactions on electron devices, 1988, vol. 35, no. 1, pp. 25-37. DOI: 10.1109/16.2412.

18. Loeffel K., Anand L. A chemo-thermo-mechanically coupled theory for elastic-viscoplastic deformation, diffusion, and volumetric swelling due to a chemical reaction. International Journal of Plasticity, 2011, vol. 27, no. 9, pp. 1409-1431. DOI: 10.1016/j.ijplas.2011.04.001.

19. Yen J.Y., Hwu J.G. Stress effect on the kinetics of silicon thermal oxidation. Journal of Applied Physics, 2001, vol. 89, no. 5, pp. 3027-3032. DOI: 10.1063/1.1342801.

20. Indeitsev D., Mochalova Y. Mechanics of multi-component media with exchange of mass and non-classical supplies. In: Dynamics of Mechanical Systems with Variable Mass, 2014, pp. $165-194$.

21. Deal B.E., Grove A.S. General relationship for the thermal oxidation of silicon. Journal of Applied Physics, 1965, vol. 36, no. 12, pp. 3770-3778.

22. Delph T.J. Intrinsic strain in SiO 2 thin films. Journal of Applied Physics, 1998, vol. 83, no. 2, pp. 786-792. DOI: 10.1063/1.366759.

23. Lin M.T. Stress Effects and Oxidant Diffusion in the Planar Oxidation. Thesis and Dissertation, Lehigh University, 1999.

24. Vilchevskaya E.N., Freidin A.B., Morozov N.F. Kinetics of the chemical reaction front in centrally symmetric problems of mechanochemistry. Doklady Physics, 2015, vol. 60, iss. 4, pp. 175-179. DOI: 10.1134/S1028335815040072.

25. Vilchevskaya E.N., Freidin A.B. On kinetics of chemical reaction fronts in elastic solids. In: Surface Effects in Solid Mechanics, Springer, Berlin, Heidelberg, 2013, pp. 181-194. 
Подана в журнал: 18.06 .2018

УДК 539.3, 544

DOI: $10.17804 / 2410-9908.2018 .6 .059-082$

\title{
ВЛИЯНИЕ ВЫБОРА МОДЕЛИ ДИФФУЗИИ НА КИНЕТИКУ ХИМИЧЕСКОЙ РЕАКЦИИ
}

\author{
П. М. Григорьева ${ }^{\text {a)* }}$ Е. Н. Вильчевская ${ }^{\text {) }}$ \\ Федеральное Государственное Бюджетное Учреждение Науки \\ Институт Проблем Машиноведения Российской Академии Наук (ИПМаш РАН), \\ Большой проспект В. О., 61, 199178, Санкт Петербург, Российская Федерация \\ a) iD https://orcid.org/0000-0001-9846-7888 polina.grigoreva239@ gmail.com; \\ б) (iD https://orcid.org/0000-0002-5173-3218 vilchevska@ gmail.com
}

\author{
* Ответственный автор. Электронная почта: polina.grigoreva239@gmail.com \\ Адрес для переписки: Большой проспект В. О., 61, 199178, Санкт-Петербург, Российская Федерация \\ Тел.: +7 (812) 321-47-78; факс: +7 (812) 321-47-71
}

Рассматривается линейно-упругое тело, в котором протекает локализированная на фронте химическая реакция, поддерживаемая диффузией газообразной компоненты через слой образующегося материала. Исследуется сравнительное влияние механических напряжений на кинетику фронта химической реакции посредством учета вклада напряжений в скорость поверхностной реакции через тензор химического сродства и в диффузионный процесс через различные зависимости коэффициента диффузии от напряжений. В качестве примера рассматривается распространение центрально-симметричного и осесимметричного фронтов реакции в различных краевых задачах при использовании разных моделей диффузии.

Ключевые слова: механохимия, тензодиффузия, неравновесная термодинамика.

\section{1. Введение}

Взаимосвязи между химическими реакциями и механическими напряжениями, возникающими в деформируемых твердых телах, представляют интерес как для фундаментальной науки, так и для инженерных приложений. В этой статье рассматривается влияние механических напряжений на кинетику химической реакции между твердой и газообразной компонентами:

$$
n_{-} B_{-}+n_{*} B_{*} \rightarrow n_{+} B_{+} \text {, }
$$

где $B_{-}$и $B_{+}$- твердые компоненты; $B_{*}$ - газовая компонента; $n_{-}, n_{+}, n_{*}$ - стехиометрические коэффициенты. Полагаем, что реакция локализована на фронте реакции $\Gamma$, которая разделяет области, занятые исходными и трансформированными материалами. Реакция поддерживается диффузией газовой компоненты $B_{*}$ извне к поверхности химической реакции сквозь образовавшийся материал. Таким образом, реакция включает два процесса - объемную диффузию газа через трансформированный материал и саму химическую реакцию. Оба процесса могут зависеть от механических напряжений. Влияние внешних и внутренних напряжений на кинетику фронта реакции было исследовано многими учеными, которые впоследствии представили различные модели. Например, Као $[1,2]$ начал моделировать напряжения в цилиндрических структурах и постулировал связь между скоростью реакции и компонентой радиального напряжения. Через год Сутарья и Олдхэм [3] представили более конкретную 
модель: они эмпирически связали нормальные напряжения $\sigma_{n n}$ и давление $p$ с двумя величинами, параметром скорости поверхностной реакции $k_{*}$ и коэффициентом диффузии $D$ в рамках экспоненциального подхода Больцмана, а именно:

$$
k_{*}=k_{0}(T) \exp \left(\frac{\sigma_{n n} V_{k}}{k T}\right), \quad D=D_{0}(T) \exp \left(-\frac{p V_{d}}{k T}\right),
$$

где $k_{0}$ и $D_{0}$ - зависящие от температуры параметр скорости поверхностной реакции и коэффициент диффузии; $k$ - постоянная Больцмана; $T$ - абсолютная температура; $V_{k}, V_{d}-$ подгоночные коэффициенты, имеющие размерность объема. Отметим, что данные зависимости параметров от напряжений были введены эвристически. Выбор параметров моделей был основан на корректировке теоретических результатов и экспериментальных данных по кинетике окисления.

Влияние механических напряжений на процесс диффузии также учитывалось через введение в уравнение диффузии дополнительных членов, зависящих от напряжений [4-8]. Эти дополнительные члены обычно зависят от изменения объема или жесткости твердого материала, либо от концентрации газа $[9,5]$. Иной подход для учета влияния напряженнодеформированного состояния на фронте химической реакции на скорость распространения фронта был представлен недавно в рамках теории рациональной механики сплошных сред [10-14]. Он основан на рациональном выводе выражения для тензора химического сродства, что позволило естественным образом получить зависимость скорости распространения от напряжений.

В данной работе исследуется сравнительное влияние напряжений на кинетику фронта химической реакции посредством учета вклада напряжений в тензор химического сродства и коэффициент диффузии. В рамках первого подхода напряжения влияют на распространение фронта химической реакции, так как они появляются в выражении для химического сродства и в соответствующем кинетическом уравнении, которое связывает химическое сродство с скоростью реакции. Второй подход сводит задачу распространения фронта химической реакции под напряжением к задаче диффузии с правильным выбором диффузионной модели и зависимыми от напряжения параметрами модели. Поскольку выражение для тензора химического сродства было получено в предположении, что трансформированный материал выступает в качестве жесткого каркаса для диффундирующего газового компонента и газ дополнительных деформаций скелета не вызывает, мы не будем рассматривать дополнительные члены в уравнении диффузии и сосредоточимся только на зависимости коэффициента диффузии от напряжений.

Обычно в моделях зависимой от напряжений диффузии используется скалярная характеристика тензора напряжений. Эта характеристика может быть определена по-разному, например, как давление, как нормальное напряжение, как интенсивность сдвиговых напряжений и др. В настоящей работе предложена связь коэффициента диффузии с тензором напряжений деформации материала, через который происходит диффузия, что приводит к модели тензорной диффузии.

Используя модель химического сродства вкупе с разными диффузионными моделями, исследуем кинетику распространения фронта химической реакции в различных краевых задачах. Распространение химической реакции при постоянном коэффициенте диффузии сравнивается с результатами, полученными при использовании эмпирического коэффициента диффузии и введенного коэффициента тензодиффузии, что позволяет исследовать, как учет зависимости диффузии от напряжений влияет на распространение фронта химической реакции, и оценить диапазон внешних нагрузок, для которых зависимостью коэффициента диффузии от напряжений можно пренебречь и считать его постоянным. 


\section{2. Постановка задачи и методы решения}

\section{1. Химическое сродство и кинетика химической реакции}

В классической химии скорость реакции определяется химическим сродством реакции, которое является комбинацией химических потенциалов, участвующих в химической реакции компонент:

$$
A=-\sum n_{k} M_{k} \mu_{k}
$$

где $\boldsymbol{\mu}_{k}$ - относительный (на единицу массы) химический потенциал $k$-й компоненты; $M_{k}-$ молярная масса, стехиометрический коэффициент $n_{k}$ входит в сумму со знаком «+», если $k$-я компонента производится в результате реакции, и со знаком «-», если расходуется. Химическое сродство широко используется в термодинамической теории химических реакций. В частности, в [16] было предложено следующее кинетическое уравнение для скорости химической реакции в зависимости от химического сродства:

$$
\boldsymbol{\omega}=k_{*} c\left\{1-\exp \left(-\frac{A}{R T}\right)\right\} \text {. }
$$

Здесь $\boldsymbol{\omega}$ - скорость химической реакции; $k_{*}$ - кинетическая константа (параметр реакции); $R=8,31$ - универсальная газовая постоянная; $T$ - температура; $c$ - молярная концентрация газовой компоненты реакции.

В случае химических реакций в газах и жидкостях, где напряжения определяются скалярной величиной - давлением, химический потенциал также является скалярной величиной. В случае твердых реагирующих компонент химический потенциал становится тензором. В результате изучения фазового равновесия было показано [10], что тензор химического потенциала для твердой компоненты определяется тензором энергии-импульса Эшелби. В работе [12] выражение для тензора химического сродства было получено как результат анализа уравнений баланса массы, импульса и энергии, а также неравенства энтропии, которое было записано для химической реакции между газовой и твердыми компонентами произвольной реологии. А именно, в диссипативном неравенстве для химической реакции было показано, что скорость реакции на ориентированной площадке с нормалью $\boldsymbol{n}$ сопряжена с нормальной компонентой $\boldsymbol{A}_{\boldsymbol{n}}=\boldsymbol{n} \cdot \boldsymbol{A} \cdot \boldsymbol{n}$ тензора $\boldsymbol{A}$, который и приняли за тензор химического сродства.

В работе [13] нормальная компонента тензора химического сродства в случае линейноупругих твердых компонент и идеального газа может быть рассчитана следующим образом:

$$
A_{n n}=\frac{n_{-} M_{-}}{\rho_{-}}\left[\gamma(T)+\frac{1}{2} \sigma_{-}: \varepsilon_{-}-\frac{1}{2} \sigma_{+}:\left(\varepsilon_{+}-\varepsilon_{c h}\right)+\frac{1}{2} \sigma_{+}:\left(\varepsilon_{+}-\varepsilon_{-}\right)\right]+n_{*} R T \ln \frac{c(\Gamma)}{c_{*}},
$$

где $M_{-}$и $\boldsymbol{\rho}_{-}-$молярная масса и плотность твердой компоненты $B_{-} ; \quad \boldsymbol{\sigma}=C_{-}: \underline{\varepsilon}$ и $\boldsymbol{\sigma}_{+}=\boldsymbol{C}_{+}:\left(\boldsymbol{\varepsilon}_{+}-\boldsymbol{\varepsilon}_{c h}\right)-$ тензоры напряжений Коши; $C_{ \pm}-$тензоры упругих модулей твердых компонент; $\varepsilon_{ \pm}-$тензоры деформации; $c(\Gamma)$ - концентрация газовой компоненты на фронте химической реакции; $c_{*}$ - любая отсчетная концентрация газовой компоненты, в данной работе за нее принята растворимость газовой компоненты в сформировавшемся материале $B_{+}$; $\varepsilon_{c h}$ - тензор деформации химических превращений. Параметр $\gamma(T)$ соответствует отсчетно- 
му уровню химических энергий при нулевых напряжениях и заданной температуре. Если температура $T$ задана, то $\gamma(T)$ является параметром модели.

Замена скалярного химического сродства нормальной компонентой тензора химического сродства в (4) и учет баланса массы на фронте химической реакции $\rho_{-} V=n_{-} M_{-} \omega$ позволяют получить следующее выражение для нормальной компоненты скорости фронта химической реакции:

$$
V=\frac{n_{-} M_{-}}{\rho_{-}} \boldsymbol{\omega}\left\{1-\exp \left(-\frac{A_{n n}}{R T}\right)\right\} .
$$

Таким образом, фронт химической реакции продвигается, только если $A_{n n}>0$. В случае, когда $A_{n n}=0$, фронт химической реакции неподвижен, что соответствует случаю химического равновесия, когда скорости прямой и обратной химической реакции совпадают. Таким образом, уравнение $A_{n n}=0$ определяет равновесную концентрацию газовой компоненты $B_{*}\left(c_{e q}\right)$ на фронте реакции в зависимости от параметров материала и напряженнодеформированного состояния на фронте реакции:

$$
\frac{n_{-} M_{-}}{\rho_{-}}\left(\gamma(T)+\frac{1}{2} \sigma_{-}: \varepsilon_{-}-\frac{1}{2} \sigma_{+}:\left(\varepsilon_{+}-\varepsilon_{c h}\right)+\sigma_{+}:\left(\varepsilon_{+}-\varepsilon_{-}\right)\right)+n_{*} R T \ln \frac{c_{e q}}{c_{*}}=0 .
$$

Ввод равновесной концентрации позволяет переписать формулу для скорости фронта химической реакции вблизи равновесия в следующем виде:

$$
V=\frac{n_{-} M_{-}}{\rho_{-}} k_{*} c(\Gamma)\left(1-\left(\frac{c_{e q}}{c(\Gamma)}\right)^{n_{*}}\right) .
$$

Таким образом, процедура определения кинетики фронта химической реакции сводится к следующему: концентрация газовой компоненты на фронте реакции $c(\Gamma)$ и равновесная концентрация $c_{e q}$ определяются из решения диффузионной задачи и уравнения (7) соответственно, после чего найденные значения подставляем в формулу для нахождения скорости фронта химической реакции (8). В итоге, интегрируя выражение для скорости фронта химической реакции по времени, находим зависимость положения фронта химической реакции от времени.

\section{2. Задача диффузии}

Концентрация газа на фронте реакции и внутри трансформированного материала будем определять согласно уравнению диффузии. Самой простой моделью диффузии является закон Фика:

$$
\frac{\partial c}{\partial t}=\nabla \cdot(D \nabla c)
$$

где $D$ - коэффициент диффузии. Мы предполагаем, что диффузионный процесс является стационарным, потому что он протекает намного быстрее, чем процесс распространения фронта химической реакции. Тогда это уравнение примет вид:

$$
\nabla \cdot(D \nabla c)=0
$$


Граничными условиями являются:

$$
D \frac{\partial c}{\partial n}+\alpha\left(c_{*}-c\right)=0 \text { на } \Omega, \quad D \frac{\partial c}{\partial n}+n_{*} k_{*} c\left(1-\left(\frac{c_{e q}}{c}\right)^{n_{*}}\right)=0 \text { на } \Gamma
$$

Первое условие следует из непрерывности потока массы на внешней поверхности тела $\Omega$. Здесь $n$ - внешняя нормаль по отношению к области $B_{+} ; \boldsymbol{\alpha}$ - поверхностная скорость переноса массы в $B_{+}$. При $\boldsymbol{\alpha}>D$ первое условие сводится к $c=c_{*}$ и становится условием заданной концентрации на внешней границе тела. Второе условие следует из баланса массы на фронте реакции $Г$. Оно означает, что весь диффузионный поток потребляется химической реакцией.

В дальнейшем будут рассмотрены три различные модели диффузии. В первой используется постоянный коэффициент диффузии. Вторая основана на широко используемой зависимости коэффициента диффузии от давления ([17-19]):

$$
D=D_{0} \exp \left(-\frac{p V_{d}}{k T}\right) ; \quad p=-\frac{1}{3}\left(\sigma_{11}^{+}+\sigma_{22}^{+}+\sigma_{33}^{+}\right) \text {. }
$$

Как упоминалось выше, это выражение носит феноменологический характер и основано на интуитивной оценке экспериментальных данных [3].

Чтобы отчетливее представлять, как напряженно-деформированное состояние твердого материала влияет на коэффициент диффузии, рассмотрим двухкомпонентную систему, состоящую из твердой и газообразной компоненты. Для простоты рассмотрим стационарную одномерную диффузию, т. е. плотность газовой составляющей и скорости зависят только от координаты оси, вдоль которой протекает диффузия: $\boldsymbol{\rho}_{g}=\boldsymbol{\rho}_{g}\left(x_{1}\right) ; \mathrm{d} u_{g} / \mathrm{d} t=V_{g}\left(x_{1}\right) ; \mathrm{d} u_{s} / \mathrm{d} t=V_{s}\left(x_{1}\right)$, где $u_{s}, V_{s}$ и $u_{g}, V_{g}$ - смещения и скорости твердых и газовых компонент соответственно, и предположим, что твердое тело является изотропным линейным упругим материалом. Тогда уравнения баланса количества движения для газообразной и твердой компонент и баланс массы для газовой компоненты будут иметь вид:

$$
\left\{\begin{array}{l}
\frac{E_{+}\left(1-\boldsymbol{v}_{+}\right)}{\left(1+\boldsymbol{v}_{+}\right)\left(1-2 \boldsymbol{v}_{+}\right)} \frac{\mathrm{d}^{2} u_{s}}{\mathrm{~d} x_{1}^{2}}+f_{s g}=0, \\
\frac{\mathrm{d} p_{g}}{\mathrm{~d} x_{1}}-f_{s g}=0, \\
\frac{\mathrm{d}\left(\boldsymbol{\rho}_{g} V_{g}\right)}{\mathrm{d} x_{1}}=0 .
\end{array}\right.
$$

Здесь $E_{+}$и $\boldsymbol{v}_{+}$- модуль Юнга и коэффициент Пуассона твердой компоненты; $f_{s g}=\rho_{g} a\left(V_{s}-V_{g}\right)-$ вязкая сила взаимодействия между газовой и твердой компонентами; $a$ - параметр вязкого трения, который может зависеть от напряженно-деформированного состояния системы.

Предположим, что газовая компонента представлена уравнением состояния идеального газа: $p_{g}=\frac{R T}{M_{g}} \rho_{g}$. Тогда решение системы (13) имеет следующий вид: 
$u_{s}=c_{1} \exp \left(-c_{2} x_{1}\right)+c_{3} x_{1}+\frac{R T}{M_{g} a} c_{2} t+c_{4}$, где $c_{1}, c_{2}, c_{3}, c_{4}-$ константы интегрирования. Откуда следует, что диффузионный поток, определяющийся соотношением $j=\boldsymbol{\rho}_{g}\left(V_{s}-V_{g}\right)$, равен $j=\frac{1}{a} \frac{E_{+}\left(1-\boldsymbol{v}_{+}\right)}{\left(1+\boldsymbol{v}_{+}\right)\left(1-2 \boldsymbol{v}_{+}\right)} c_{1} c_{2}^{2} \exp \left(-c_{2} x_{1}\right)$. Учитывая, что концентрация газа равна $c=\boldsymbol{\rho}_{g} / M_{g}$, можно заметить, что диффузионный поток пропорционален градиенту концентрации $\left(j=\frac{R T}{a} \frac{\mathrm{d} c}{\mathrm{~d} x_{1}}\right)$, что приводит к закону Фика, в котором коэффициентом диффузии является $\frac{R T}{a}$. Таким образом, в рамках рассмотренной модели напряженно-деформированное состояние твердой компоненты может влиять на диффузию только через параметр $a$. Схожие результаты были получены в [20].

В большинстве случаев диффузия является объемной, и в этом случае диффузионный поток двигается в пространстве между узлами решетки твердого тела. Параметр $a$ определяет, как легко молекулы газа могут диффундировать, и, следовательно, зависит от пространства между узлами решетки. Для того, чтобы рассмотреть зависимость диффузии от механических нагрузок, примем, что параметр $a$ находится в обратной зависимости от деформаций, происходящих в плоскости, перпендикулярной диффузионному потоку. Другими словами, мы считаем, что деформации вдоль направления диффузионного потока $\left(\varepsilon_{11}\right)$ не влияют на диффузию. Тогда коэффициент диффузии в направлении $x_{1}$ выглядит следующим образом:

$$
D=D_{0}\left(1+\beta\left(\varepsilon_{22}+\varepsilon_{33}\right)\right)
$$

где $\beta$ - постоянный параметр.

Следовательно, в трехмерном случае мы приходим к модели тензорной диффузии, в которой коэффициент диффузии имеет различное значение в разных направлениях:

$$
\boldsymbol{D}=D_{0}\left[\begin{array}{ccc}
1+\boldsymbol{\beta}_{1}\left(\boldsymbol{\varepsilon}_{22}+\boldsymbol{\varepsilon}_{33}\right) & 0 & 0 \\
0 & 1+\boldsymbol{\beta}_{2}\left(\boldsymbol{\varepsilon}_{11}+\boldsymbol{\varepsilon}_{33}\right) & 0 \\
0 & 0 & 1+\boldsymbol{\beta}_{3}\left(\boldsymbol{\varepsilon}_{22}+\boldsymbol{\varepsilon}_{11}\right)
\end{array}\right] .
$$

Для того чтобы сравнить кинетику фронта химической реакции с коэффициентами диффузии, заданными по формулам (12) и (15), нужно оценить параметр $\beta$. Разложив в ряд коэффициент диффузии (12), переписав его через деформации при помощи законом Гука и сравнив результат с коэффициентом диффузии (15), мы увидим, что эти два подхода согласуются, если параметр $\beta$ равен $\boldsymbol{\beta}_{*}=\frac{V_{d} E_{+}}{\left(1-2 \boldsymbol{v}_{+}\right) T k}$.

Обратим внимание, что в целом эти два подхода дают разные результаты. Например, в случае одноосного растяжения в направлении диффузионного потока феноменологический подход (12) приводит к увеличению скорости диффузии, а тензорная диффузия (15) приведет к замедлению диффузии, поскольку межмолекулярное пространство в плоскости, перпендикулярной диффузионному потоку, уменьшится из-за эффекта Пуассона. 


\section{3. Аналитическое решение некоторых краевых задач}

\subsection{1. Центрально-симметричное тело}

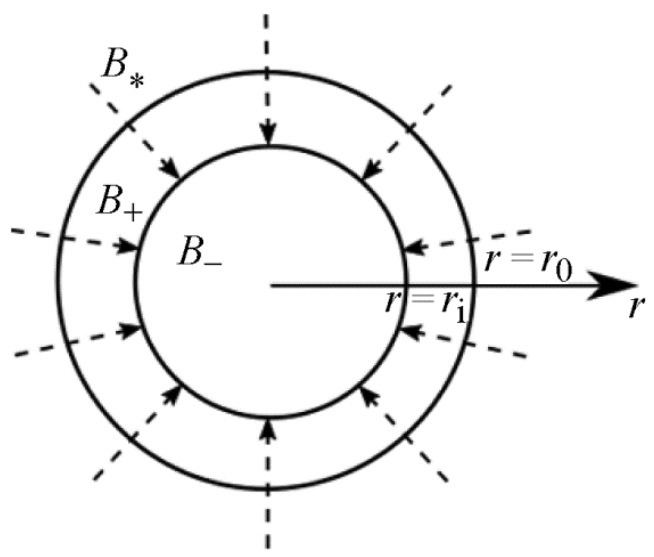

Рис. 1. Центрально-симметричное тело

Рассмотрим тело со сферической симметрией $r \in\left[0, r_{\mathrm{o}}\right]$, в котором химическая реакция распространяется с поверхности тела противоположно направлению оси $r$ и локализована на сферической поверхности с координатами $r=r_{\text {i }}$ (рис. 1). Тензор химических превращений - изотропный $\boldsymbol{\varepsilon}_{\boldsymbol{h}}=\boldsymbol{\varepsilon}_{c h} \boldsymbol{E}$, где $\boldsymbol{E}-$ единичный тензор. Будем рассматривать два типа граничных условий: на внешней поверхности тела заданы радиальные перемещения $u_{0}$ или давление $p_{0}$. Из соображений симметрии примем, что концентрация газовой компоненты $B_{*}$ не зависит от координат $\vartheta$ и $\boldsymbol{\varphi}$, т. е. является функцией только радиальной координаты, $c=c(r)$.

Перемещения в теле могут быть найдены из решения Ламе для тел со сферической симметрией:

$$
u_{r \pm}=A_{ \pm} r+\frac{B_{ \pm}}{r^{2}}
$$

где константы $A_{ \pm}$и $B_{ \pm}$определяются из условий непрерывности радиальных компонент перемещений и напряжений $\left(\sigma_{r r}\right)$ на фронте химической реакции и ограниченностью перемещений в центре шара при $r=0$. Следовательно, с учетом этих условий перемещения будут выглядеть следующим образом:

$$
u_{-}=A_{-} r, u_{+}=A_{+} r+\frac{\left(A_{-}-A_{+}\right) r_{i}^{3}}{r^{2}},
$$

где:

$$
A_{+}=\frac{A_{-}\left(3 k_{-}+4 \mu_{+}\right)+3 k_{+} \varepsilon_{\mathrm{ch}}}{3\left(\lambda_{+}+2 \mu_{+}\right)} .
$$


Здесь $k_{ \pm}$- модули всестороннего сжатия; $\lambda_{ \pm}-$параметры Ламе; $\boldsymbol{\mu}_{ \pm}-$модули сдвига компонент $B_{-}$и $B_{+}$соответственно. Константа $A_{-}$зависит от типа граничных условий и для случая заданных на поверхности перемещений $u_{0}$ имеет вид:

$$
A_{-}=\frac{\left(\lambda_{+}+2 \mu_{+}\right) 3 \overline{u_{0}}-3 k_{+}\left(1-\xi^{3}\right) \varepsilon_{\mathrm{ch}}}{3 k_{-}+4 \mu_{+}+3\left(k_{+}-k_{-}\right) \xi^{3}}, \quad \overline{u_{0}}=\frac{u_{0}}{r_{\mathrm{o}}}
$$

и

$$
A_{-}=\frac{\left(\lambda_{+}+2 \mu_{+}\right) p_{0}+4 k_{+} \mu_{+}\left(1-\xi^{3}\right) \boldsymbol{\varepsilon}_{c h}}{k_{+}\left(3 k_{-}+4 \boldsymbol{\mu}_{+}\right)-4 \mu_{+}\left(k_{+}-k_{-}\right) \xi^{3}}
$$

в случае заданных напряжений. Здесь и далее используется безразмерный параметр $\xi=\frac{r_{\mathrm{i}}}{r_{\mathrm{o}}}$.

Тогда компоненты деформаций и напряжений имеют следующий вид:

$$
\begin{aligned}
& \boldsymbol{\varepsilon}_{r r}^{-}=\boldsymbol{\varepsilon}_{\varphi \varphi}^{-}=\boldsymbol{\varepsilon}_{\theta \theta}^{-}=A_{-} ; \quad \sigma_{r r}^{-}=\sigma_{\varphi \varphi}^{-}=\sigma_{\theta \theta}^{-}=3 k_{-} A_{-} ; \\
& \boldsymbol{\varepsilon}_{r r}^{+}=A_{+}-2 \frac{\left(A_{-}-A_{+}\right) r_{\mathrm{i}}^{3}}{r^{3}} ; \quad \boldsymbol{\varepsilon}_{\varphi \varphi}^{+}=\boldsymbol{\varepsilon}_{\theta \theta}^{+}=A_{+}+\frac{\left(A_{-}-A_{+}\right) r_{\mathrm{i}}^{3}}{r^{3}} ; \\
& \boldsymbol{\sigma}_{r r}^{+}=3 \lambda_{+} A_{+}-4 \mu_{+} \frac{\left(A_{-}-A_{+}\right) r_{\mathrm{i}}^{3}}{r^{3}}-3 k_{+} \boldsymbol{\varepsilon}_{c h} ; \\
& \sigma_{\varphi \varphi}^{+}=\sigma_{\theta \theta}^{+}=3 \lambda_{+} A_{+}+2 \mu_{+} \frac{\left(A_{-}-A_{+}\right) r_{\mathrm{i}}^{3}}{r^{3}}-3 k_{+} \boldsymbol{\varepsilon}_{c h} .
\end{aligned}
$$

Окончательно уравнение для определения равновесной концентрации будет выглядеть следующим образом:

$$
\begin{aligned}
& A_{n n}=\frac{n_{-} M_{-}}{\rho_{-}}\left[\gamma(T)+\frac{1}{2\left(\lambda_{+}+2 \mu_{+}\right)}\left(12\left(k_{-}-k_{+}\right) \mu_{+} A_{-}^{2}+3 k_{+}\left(3 k_{-}+8 \mu_{+}\right) A_{-} \boldsymbol{\varepsilon}_{c h}\right.\right. \\
& \left.\left.-12 \boldsymbol{\mu}_{+} k_{+} \boldsymbol{\varepsilon}_{c h}^{2}\right)\right]+n_{*} R T \ln \left(\frac{c(\boldsymbol{\xi})}{c_{*}}\right)=0
\end{aligned}
$$

Как упоминалось, реакция может протекать только при $A_{n n}>0$. Таким образом, при отсутствии внешних нагрузок и нулевой толщине превращенного слоя реакция может начаться только в том случае, когда величина энергетического параметра больше некоторого критического значения $\left(\gamma_{*}\right)$, зависящего от свойств материала:

$$
\boldsymbol{\gamma}>\boldsymbol{\gamma}_{*}=\frac{E_{+}}{1-\boldsymbol{v}_{+}} \boldsymbol{\varepsilon}_{c h}^{2}
$$

Также отметим, что рост слоя превращенного материала инициирует возникновение внутренних напряжений, порожденных деформацией химического превращения, что, при небольших значениях $\gamma$, может заблокировать дальнейшее протекание реакции. 
Уравнение диффузии в сферических координатах выглядит следующим образом:

$$
D \frac{\mathrm{d}^{2} c}{\mathrm{~d} \zeta^{2}}+\left(\frac{\mathrm{d} D}{\mathrm{~d} \zeta}+\frac{2}{\zeta} D\right) \frac{\mathrm{d} c}{\mathrm{~d} \zeta}=0, \quad \zeta=\frac{r}{r_{\mathrm{o}}}
$$

Заметим, что в данной краевой задаче след тензора напряжений не зависит от положения фронта химической реакции:

$$
\operatorname{tr} \sigma^{+}=\sigma_{r}^{+}+\sigma_{\varphi}^{+}+\sigma_{\vartheta}^{+}=3 k_{+}\left(A_{+}-\varepsilon_{\mathrm{ch}}\right),
$$

поскольку $A_{+}$не зависит от $r$ и, соответственно, не зависит от $\boldsymbol{\zeta}=r / r_{\mathrm{o}}$. Таким образом, в случае постоянного коэффициента диффузии и коэффициента диффузии определенного соотношением (12) $\left(\frac{\mathrm{d} D}{\mathrm{~d} \zeta}=0\right)$ и, следовательно, в обоих случаях концентрация газа на фронте реакции определяется уравнением:

$$
\frac{\mathrm{d}^{2} c}{\mathrm{~d} \zeta^{2}}+\frac{2}{\zeta} \frac{\mathrm{d} c}{\mathrm{~d} \zeta}=0
$$

Решением этого уравнения будет $c=\frac{c_{1}}{\zeta}+c_{2}$. Граничные условия (11) для нахождения констант интегрирования в этом случае будут выглядеть следующим образом:

$$
-\frac{D}{r_{\mathrm{o}}} c_{1}+\alpha\left(c_{*}-c_{1}-c_{2}\right)=0, \quad-\frac{D}{r_{\mathrm{o}}} \frac{c_{1}}{\xi^{2}}+n_{*}^{2} k_{*}\left(\frac{c_{1}}{\xi}+c_{2}-c_{\mathrm{eq}}\right)=0 .
$$

Подставив найденную равновесную концентрацию и концентрацию газа на фронте реакции в (8) и введя безразмерные параметры

$$
t_{*}=\frac{n_{-} k_{*} n_{*} M_{-} c_{*}}{r_{\mathrm{o}} \rho_{-}} t, \boldsymbol{\kappa}_{1}=\frac{n_{*}^{2} k_{*} r_{\mathrm{o}}}{D_{0}}, \boldsymbol{\kappa}_{2}=\frac{n_{*}^{2} k_{*}}{\boldsymbol{\alpha}},
$$

получим следующее уравнение, описывающее скорость распространения фронта химической реакции

$$
\frac{d \xi}{d t_{*}}=\frac{1-c_{\mathrm{eq}} / c_{*}}{1+\kappa_{1} \xi-\kappa_{1} \xi^{2}+\kappa_{2} \xi^{2}}
$$

для постоянного коэффициента диффузии и

$$
\frac{d \xi}{d t_{*}}=\frac{1-c_{\mathrm{eq}} / c_{*}}{1+\frac{\boldsymbol{\kappa}_{1}}{\overline{\bar{B}} \boldsymbol{\xi}}-\frac{\boldsymbol{\kappa}_{1}}{\overline{\bar{B}}} \xi^{2}+\boldsymbol{\kappa}_{2} \xi^{2}}
$$


для эмпирического коэффициента диффузии (12), где $\overline{\bar{B}}=\exp \left(\frac{V_{d}}{k T} 3 k_{+}\left(A^{+}-\varepsilon_{\text {ch }}\right)\right)$. Замена $\tilde{\xi}=1-\xi$ даст нам зависимость скорости роста относительной толщины превращенного слоя от времени.

Рассмотрим коэффициент диффузии (14), зависящий от деформаций скелета. В случае сферического тела этот коэффициент будет равен $D=D_{0}\left(1+\boldsymbol{\beta}\left(\boldsymbol{\varepsilon}_{\varphi \varphi}^{+}+\boldsymbol{\varepsilon}_{\theta \theta}^{+}\right)\right)$. С учетом формул (21) уравнение диффузии может быть записано как

$$
\left(1+2 \beta\left(A_{+}+\frac{B \xi^{3}}{\zeta^{3}}\right)\right) \frac{\mathrm{d}^{2} c}{\mathrm{~d} \zeta^{2}}+\frac{2\left(\zeta^{3}\left(1+2 A_{+} \boldsymbol{\beta}\right)-B \boldsymbol{\beta} \xi^{3}\right)}{\zeta^{4}} \frac{\mathrm{d} c}{\mathrm{~d} \zeta}=0 .
$$

Решение этого уравнения имеет следующий вид:

$$
c(\zeta)=\frac{C_{1}\left(2 \sqrt{3} \tan ^{-1}(\psi)-3 \ln \left(p^{1 / 3} \zeta+q^{1 / 3} \xi\right)+\ln \left(p \zeta^{3}+q \xi^{3}\right)\right)}{6 p^{2 / 3} q^{1 / 3} \xi}+C_{2},
$$

где $p=1+2 \beta A_{+} ; q=2 B \boldsymbol{\beta} ; \psi=\frac{-1+\frac{2 p^{1 / 3} \zeta}{q^{1 / 3} \xi}}{\sqrt{3}}$. Константы интегрирования $C_{1}$ и $C_{2}$ находятся из граничных условий (11).

Разложив уравнение (8) в ряд около $\boldsymbol{\xi}=1$ и учитывая, что $p+q=1+2 \boldsymbol{\beta} A_{+}+$ $+2 \boldsymbol{\beta}\left(A_{-}-A_{+}\right)=1+2 A_{-} \boldsymbol{\beta}$, получаем:

$$
\frac{d \xi}{d t_{*}}=\frac{\left(1+2 A_{-} \beta\right)\left(1-c_{\mathrm{eq}} / c_{*}\right)}{\left(1+2 A_{-} \beta\right)\left(1+\kappa_{2} \xi^{2}\right)+\kappa_{1} \xi-\kappa_{1} \xi^{2}} .
$$

Заменяя $\xi$ на $\tilde{\xi}=1-\xi$, мы получим зависимость скорости роста относительной толщины превращенного слоя от времени.

\subsection{2. Осесимметричное тело}

Рассмотрим бесконечный цилиндр $r \in\left[0, r_{\mathrm{o}}\right]$, состоящий из двух слоев «+» и «-», разделенных цилиндрическим фронтом химической реакции $r=r_{\mathrm{i}}$. Примем, что реакция распространяется от внешней поверхности к центру цилиндра. На боковой поверхности цилин-

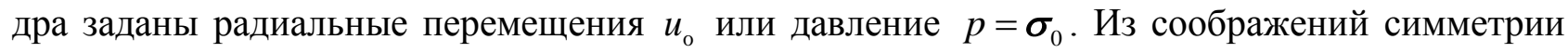
следует, что концентрация является функцией только $r$-координаты: $c=c(r)$. Примем гипотезу о плоско-деформированном состоянии, считая, что отсутствуют какие-либо деформации по оси $z$ и тензор деформаций химических превращений является плоским: $\varepsilon_{h}=\varepsilon_{\mathrm{ch}} \boldsymbol{e}_{r} \boldsymbol{e}_{\boldsymbol{r}}+\varepsilon_{\mathrm{ch}} \boldsymbol{e}_{\phi} \boldsymbol{e}_{\phi}$

Перемещения могут быть найдены из задачи Ламе:

$$
u_{r \pm}=A_{ \pm} r+\frac{B_{ \pm}}{r}
$$


С учетом непрерывности перемещений и их ограниченности в центре цилиндра, а также с учетом непрерывности радиального вектора усилий, перемещения во внутреннем и внешних областях определяются формулами:

$$
u_{-}=A_{-} r ; u_{+}=A_{+} r+\frac{\left(A_{-}-A_{+}\right)}{r},
$$

где

$$
A_{+}=\frac{\bar{k}_{-}+2 \mu_{+}}{\bar{k}_{+}+2 \mu_{+}} A_{-}+\frac{\bar{k}_{+}}{\bar{k}_{+}+2 \mu_{+}} \boldsymbol{\varepsilon}_{c h} .
$$

Здесь $\bar{k}_{ \pm}=\left(\lambda_{ \pm}+\mu_{ \pm}\right)-$плоские модули всестороннего сжатия.

Параметр $A_{-}$находим из граничных условий

$$
A_{-}=\frac{\bar{u}_{0}\left(\bar{k}_{+}+2 \mu_{+}\right)-\bar{k}_{+} \varepsilon_{\mathrm{ch}}\left(1-\xi^{2}\right)}{\bar{k}_{-}+2 \mu_{+}+\left(\bar{k}_{+}-\bar{k}_{-}\right) \xi^{2}}, \quad \bar{u}_{0}=\frac{u_{\mathrm{o}}}{r_{\mathrm{o}}}
$$

если на поверхности цилиндра заданы перемещения, и

$$
A_{-}=\frac{\overline{\sigma_{0}}+2 \varepsilon_{\mathrm{ch}}\left(1-\xi^{2}\right)}{\frac{\bar{k}_{-}}{\mu_{+}}+\frac{\bar{k}_{-}}{\bar{k}_{+}} \xi^{2}+2\left(1-\xi^{2}\right)}, \quad \overline{\sigma_{0}}=\frac{\sigma_{0}\left(\bar{k}_{+}+2 \mu_{+}\right)}{\bar{k}_{+} \mu_{+}},
$$

если на поверхности задано давление. Напомним, что параметр $\xi=\frac{r_{\text {i }}}{r_{\text {o }}}$ соответствует относительному положению фронта химической реакции. Таким образом, уравнение для равновесной концентрации имеет вид:

$$
\frac{M_{-}}{\rho_{-}}\left(\gamma(T)+\frac{1}{\bar{k}_{+}+2 \mu_{+}}\left[2\left(\bar{k}_{-}-\bar{k}_{+}\right) \boldsymbol{\mu}_{+} A_{-}^{2}+\bar{k}_{+}\left(\bar{k}_{-}+4 \mu_{+}\right) A_{-} \boldsymbol{\varepsilon}_{c h}-2 \bar{k}_{+} \boldsymbol{\mu}_{+} \boldsymbol{\varepsilon}_{c h}^{2}\right]\right)+R T \ln \frac{c(\xi)}{\tilde{n}_{*}}=0 .
$$

Реакция распространяется, если $A_{n n}>0$. Следовательно, в отсутствие внешних нагрузок и нулевой толщине превращенного слоя реакция будет идти, только если энергетический параметр будет достаточно большим:

$$
\gamma>\gamma_{*}=\frac{2 \bar{k}_{+} \mu_{+}}{\bar{k}_{+}+2 \mu_{+}} \varepsilon_{c h}^{2} .
$$

Отметим, что в этом случае критическое значение энергетического параметра $\boldsymbol{\gamma}_{*}$ в $2 / 3$ меньше, чем аналогичная величина для случая сферического тела.

Уравнение диффузии в цилиндрических координатах выглядит следующим образом:

$$
D \frac{d^{2} c}{d \zeta^{2}}+\left(\frac{d D}{d \zeta}+\frac{1}{\zeta} D\right) \frac{d c}{d \zeta}=0
$$


где $\zeta=r / r_{\text {. }}$. Внешняя поверхность $\Omega$ в этом случае будет соответствовать $\boldsymbol{\zeta}=1$, а поверхность фронта химической реакции $\Gamma-\boldsymbol{\zeta}=\boldsymbol{\xi}$.

Аналогично случаю центрально симметричного тела след тензора напряжений не зависит от толщины превращенного слоя

$$
\operatorname{Tr} \sigma^{+}=\sigma_{r}^{+}+\sigma_{\varphi}^{+}+\sigma_{z}^{+}=\left(3 \bar{k}_{+}-2 \mu_{+}\right)\left(A_{+}-\varepsilon_{\mathrm{ch}}\right),
$$

поскольку $A_{+}$не зависит от $r$ и, следовательно, коэффициент диффузии (12) также не зависит от $\boldsymbol{\zeta}$. Таким образом, для постоянного коэффициента диффузии и для феноменологического коэффициента диффузии (12) уравнение диффузии имеет вид:

$$
\frac{\mathrm{d}^{2} c}{\mathrm{~d} \zeta^{2}}+\frac{1}{\zeta} \frac{\mathrm{d} c}{\mathrm{~d} \zeta}=0
$$

Решением этого уравнения является $c=c_{1} \ln (\zeta)+c_{2}$. Константы интегрирования могут быть найдены из граничных условий (11).

Переходя к уже введенным безразмерным параметрам (28), получим уравнение для распространения относительного положения фронта химической реакции

$$
\frac{\mathrm{d} \xi}{\mathrm{d} t_{*}}=\frac{1-c_{\mathrm{eq}} / c_{*}}{1+\kappa_{1} \xi \ln \xi-\kappa_{2} \xi}
$$

для постоянного коэффициента диффузии и

$$
\frac{\mathrm{d} \xi}{\mathrm{d} t_{*}}=\frac{1-c_{\mathrm{eq}} / c_{*}}{1+\frac{\kappa_{1}}{\bar{B}} \xi \ln \xi-\kappa_{2} \xi}
$$

для эмпирического коэффициента диффузии (12), где $\bar{B}=\exp \left(\frac{V_{d}}{k T}\left(3 \bar{k}_{+}-2 \boldsymbol{\mu}_{+}\right)\left(A_{+}-\boldsymbol{\varepsilon}_{c h}\right)\right)$. Подстановка $\tilde{\xi}=1-\xi$ ведет к уравнению роста относительной толщины трансформированного слоя.

Обратимся теперь к тензорному коэффициенту диффузии (14), который в этом случае будет равен $D=D_{0}\left(1+\beta \varepsilon_{\varphi \varphi}^{+}\right)$. Учитывая, что $\varepsilon_{\varphi \varphi}^{+}=A_{+}+\left(A_{-}-A_{+}\right) \frac{\xi^{2}}{r^{2}}$, дифференциальное уравнение для концентрации принимает вид:

$$
\left(1+\boldsymbol{\beta}\left(A^{+}+\frac{B \xi^{2}}{\zeta^{2}}\right)\right) \frac{\mathrm{d}^{2} c}{\mathrm{~d} \zeta^{2}}+\frac{\zeta^{2}+A^{+} \boldsymbol{\zeta}^{2} \boldsymbol{\beta}-B \boldsymbol{\beta} \boldsymbol{\xi}^{2}}{\zeta^{3}} \frac{\mathrm{d} c}{\mathrm{~d} \zeta}=0,
$$

где $B=A_{-}-A_{+}$. Решение этого уравнения громоздко и здесь не представлено, однако, подставляя граничные условия и переходя к безразмерным параметрам (28), мы получаем следующее уравнение, описывающее кинетику относительного положения фронта химической реакции:

$$
\frac{\mathrm{d} \xi}{\mathrm{d} t_{*}}=\frac{1-c_{\mathrm{eq}} / c_{*}}{1+\kappa_{1} \ln \left(\lambda \xi^{2}\right)-\kappa_{2} \lambda \xi}
$$


Здесь $\lambda=\frac{1+A_{-} \beta}{1+A_{+} \beta+B \beta \xi^{2}}$. После подстановки $\tilde{\xi}=1-\xi$ мы получаем уравнение роста относительной толщины трансформированного слоя.

\section{3. Результаты и обсуждение}

Дальнейшие вычисления будут проводиться с параметрами, приведенными в табл. 1. Величины параметров соответствуют физическим параметрам $\mathrm{Si}$ и $\mathrm{SiO}_{2}$ за исключением химической деформации превращения $\boldsymbol{\varepsilon}_{c h}$, которая на порядок меньше величины, соответствующей изменению объема при химическом превращении кремния в диоксид кремния. Такой выбор значения $\boldsymbol{\varepsilon}_{c h}$ соответствует приближению малых деформаций и оправдан быстрой перестройкой деформаций, обеспечивающей релаксацию напряжений вследствие вязкости диоксида кремния. Значения $k_{*}, \alpha$ были взяты из [21]. Значение коэффициента диффузии $D_{0}$ различно в разных работах: в частности в работах [22] и [23] оно на три порядка выше значения, приведенного в [21]. Такой разброс значений может быть объяснен неопределенностью в определении коэффициента диффузии $D_{0}$ в теоретических и экспериментальных исследованиях, а также путаницей в использовании $D_{0}$ или зависящего от температуры коэффициента $\frac{D_{0}}{k T}$, как это произошло, например, в работе [10]. Так как численные вычисления проводятся для того чтобы продемонстрировать возможности модели и влияние диффузии под механическими напряжениями на скорость химической реакции, приведенные в табл. 1 параметры можно рассматривать как параметры искусственного материала.

Таблица 1 - Параметры материалов

\begin{tabular}{|l|c|c|}
\hline \multicolumn{1}{|c|}{ Параметры } & Постоянная $B_{-}$ & Постоянная $B_{+}$ \\
\hline Модули Юнга $E$, ППа & 163 & 60 \\
\hline Коэффициенты Пуассона, $\boldsymbol{v}$ & 0,23 & 0,17 \\
\hline Деформация химических превращений, $\boldsymbol{\varepsilon}_{c h}$ & - & 1173 \\
\hline Температура $T, \mathrm{~K}$ & \multicolumn{2}{|c|}{$6,61 \mathrm{e}-14$} \\
\hline Коэффициент диффузии $D_{0}, \mathrm{~m}^{2} / \mathrm{c}$ & \multicolumn{2}{|c|}{$0,36 \mathrm{e}-6$} \\
\hline Кинетическая константа реакции $k_{*}, \mathrm{~m} / \mathrm{c}$ & \multicolumn{2}{|c|}{0,028} \\
\hline $\begin{array}{l}\text { Скорость растворимости молекул газа } \\
\text { в новом материале } \alpha, \mathrm{m} / \mathrm{c}\end{array}$ & \multicolumn{2}{|c|}{} \\
\hline
\end{tabular}

\section{1. Кинетика фронта реакции в центрально-симметричном теле}

Подробное исследование кинетики фронта химической реакции с постоянным коэффициентом диффузии приведено в работе [24]. В нашей работе мы будем сравнивать, как влияет учет зависимости коэффициента диффузии от напряжений на скорость распространения химического фронта.

Введем относительное расхождение между положениями фронта химической реакции при использовании различных моделей диффузии:

$$
\Delta=\frac{r_{i}\left(D_{0}\right)-r_{i}(D)}{r_{0}} \cdot 100 \%=\left(\xi(D)-\xi\left(D_{0}\right)\right) \cdot 100 \%
$$




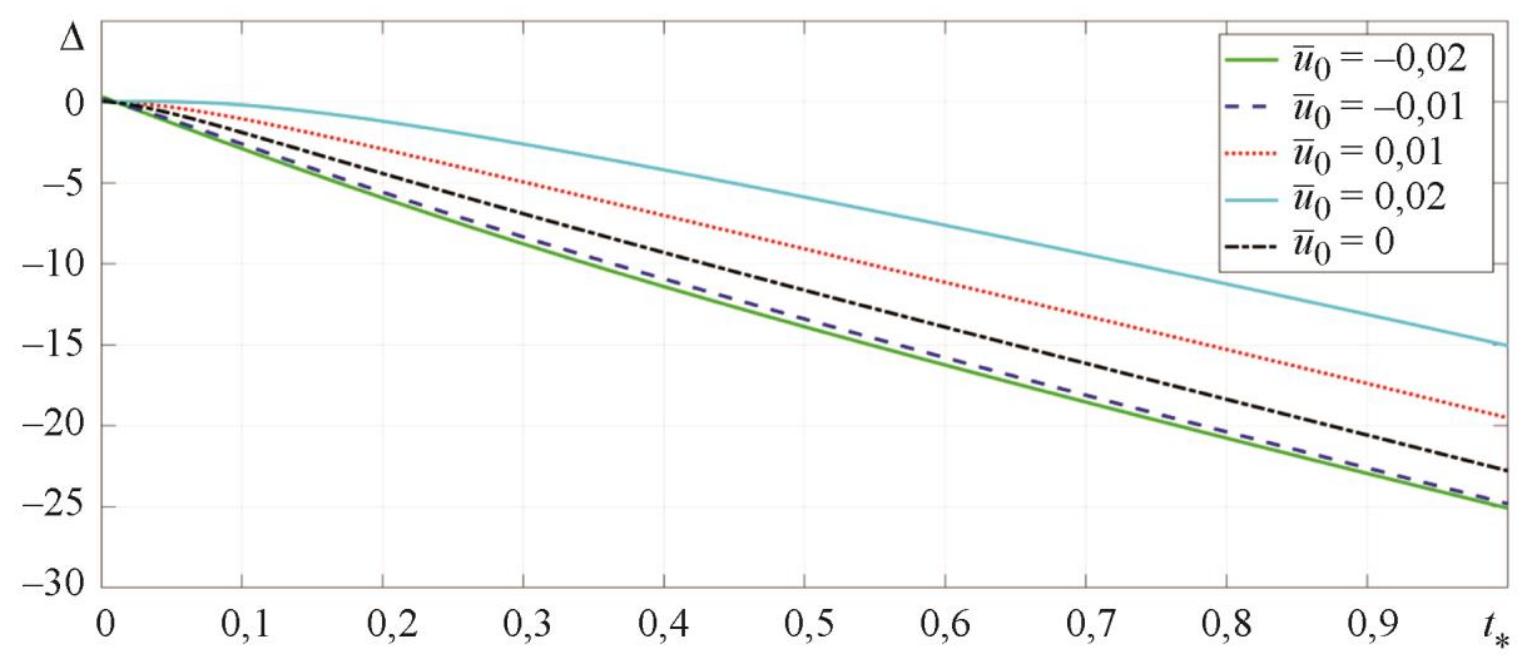

Рис. 2. Зависимость относительной разницы $\Delta$ от времени $t_{*}$ для сферического тела,

$$
\gamma=5 \gamma_{*} ; \text { заданные перемещения }
$$

Отметим, что параметр $\Delta$ характеризует не относительную погрешность межу различными моделями диффузии, а оценивает, насколько реакция замедляется или ускоряется при учете зависимости коэффициента диффузии от напряжений по сравнению с постоянным коэффициентом диффузии. Положительные значения $\Delta$ означают, что учет напряжений в диффузии ускоряет распространение фронта реакции, а отрицательные, напротив, замедляет.

Рассмотрим сначала коэффициент диффузии, определяемый соотношением (12), и случай заданных на границе перемещений $u_{0}$. На рис. 2 представлено изменение параметра $\Delta$ с течением времени при различных значениях $\overline{u_{0}}=u_{0} / r_{0}$. Учет напряжений в коэффициенте диффузии значительно замедляет реакцию, вследствие значительных сжимающих напряжений, возникающих в шаре из-за появления слоя превращенного материала с положительной собственной деформацией, в независимости от того растягивающие или сжимающие перемещения заданы на границе.

Рассмотрим теперь случай заданных напряжений. Учет коэффициента диффузии, зависящего от напряжений, как и в случае заданных перемещений, замедляет рост превращенного слоя (рис. 3). При сжимающих напряжениях разница максимальна, при растягивающих - минимальна. Однако разница между подходами в случае заданных напряжений меньше, так как задавая напряжения, мы не фиксируем границы тела и оно может свободно расширяться вследствие химических превращений, не создавая дополнительных внутренних напряжений. Тем не менее эта разница существенна даже для растягивающих напряжений, поэтому при выбранном коэффициенте диффузии в данной краевой задаче пренебречь зависимостью коэффициента диффузии нельзя даже для того, чтобы получить оценочные результаты.

Рассмотрим также влияние коэффициента диффузии (14), зависящего от деформаций скелета превращенного материала, на распространение фронта реакции. Сравнение всех трех моделей диффузии при заданных на границе перемещениях приведено на рис. 4. В отличие от феноменологического коэффициента диффузии учет коэффициента тензодиффузии может, как ускорять рост слоя превращенного материала при растягивающих перемещениях (рис. 4 a), так и замедлять их при сжимающих перемещениях (рис. 4 б). Такое существенное различие между различными коэффициентами диффузии связано прежде всего с тем, что в этой задаче наибольший вклад в феноменологический коэффициент диффузии вносит радиальная компонента напряжений, действующая по оси диффузионного потока и слабо влияющая на деформации в превращенном материале в перпендикулярной направлению диффузии плоскости. 


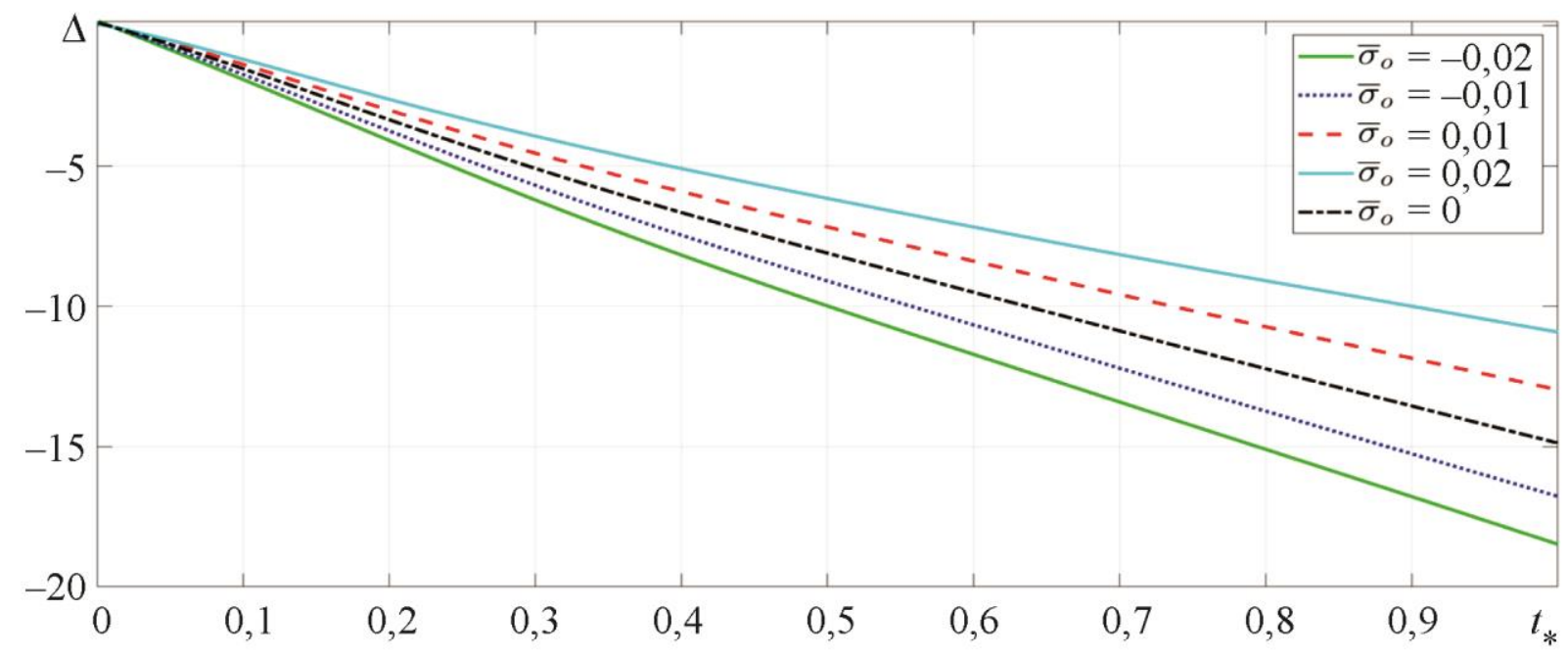

Рис. 3. Зависимость относительной разницы $\Delta$ от времени $t_{*}$ для сферического тела, $\gamma=5 \gamma_{*} ;$ заданные напряжения
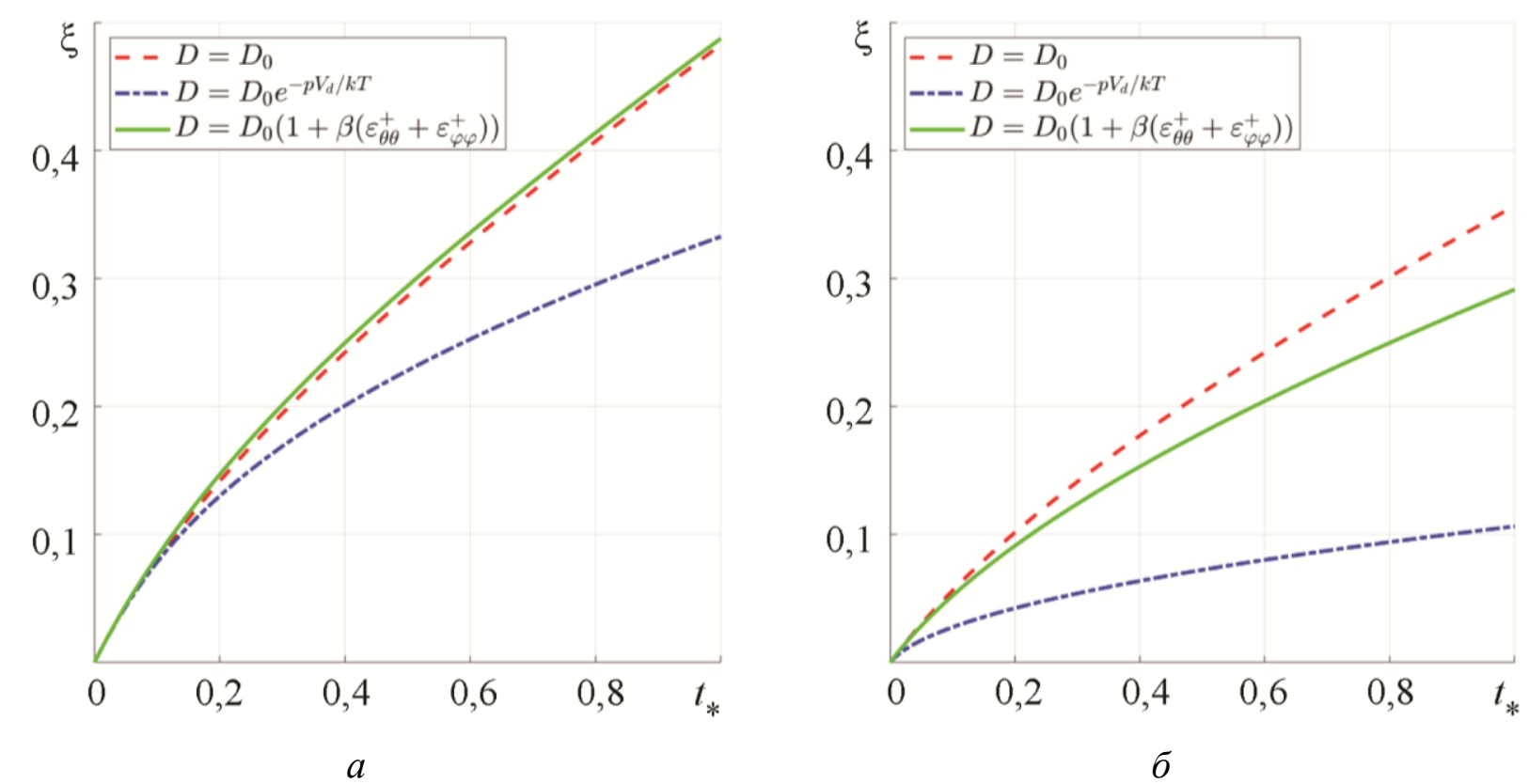

Рис. 4. Зависимость относительной толщины $\xi$ от времени $t_{*}$ для сферического тела, $\boldsymbol{\beta}=\boldsymbol{\beta}_{*}, \boldsymbol{\gamma}=5 \boldsymbol{\gamma}_{*}$; заданные перемещения на растяжение (a) и сжатие (б)

Более наглядно различие между выбором зависимости коэффициента диффузии от напряжений можно увидеть, сравнивая относительную разницу роста слоя превращенного материала при обоих подходах (рис. 5). Вне зависимости от знака перемещений на границе относительная разница между кинетикой фронта реакции с коэффициентом диффузии (14) и кинетикой с постоянным коэффициентом диффузии не превышает 10 \%. Таким образом, в данной задаче зависимость коэффициента диффузии от напряжений в виде (14) оказывает значительно меньшее влияние на кинетику распространения фронта химической реакции, чем зависимость от напряжений химического сродства. Следовательно, в этом случае можно не учитывать зависимость коэффициента диффузии от механических напряжений при проведении расчетов. 


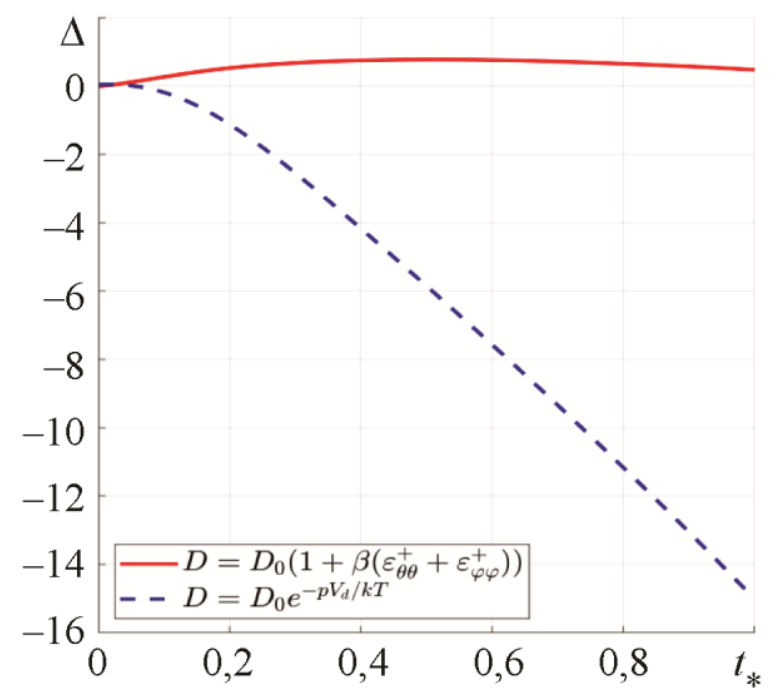

$a$

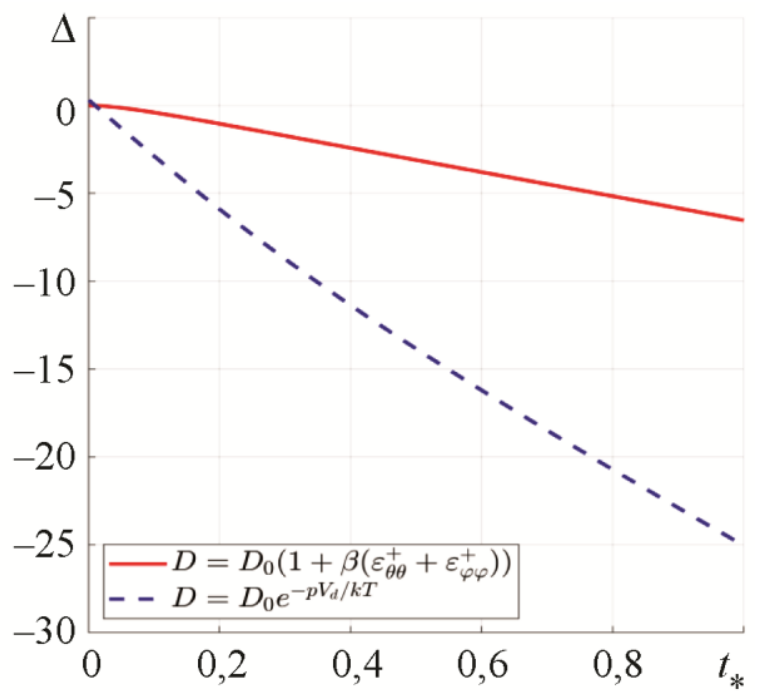

$\sigma$

Рис. 5. Зависимость относительной разницы $\Delta$ от времени $t_{*}$ для сферического тела, заданные перемещения на растяжение (a) и сжатие (б)

Если на границе шара задано давление $p_{0}$, то независимо от величины и знака $p_{0}$ учет коэффициента диффузии (14), зависящего от деформаций каркаса, ускоряет продвижение фронта химической реакции. При этом относительная разница между кинетиками фронта химической реакции с коэффициентом диффузии (14) и с постоянным коэффициентом диффузии не превышает $5 \%$, поэтому при учете зависимости диффузии от напряжений в виде тензодиффузии этой разницей можно пренебречь и не учитывать зависимость коэффициента диффузии от механических напряжений без существенного снижения точности вычислений.

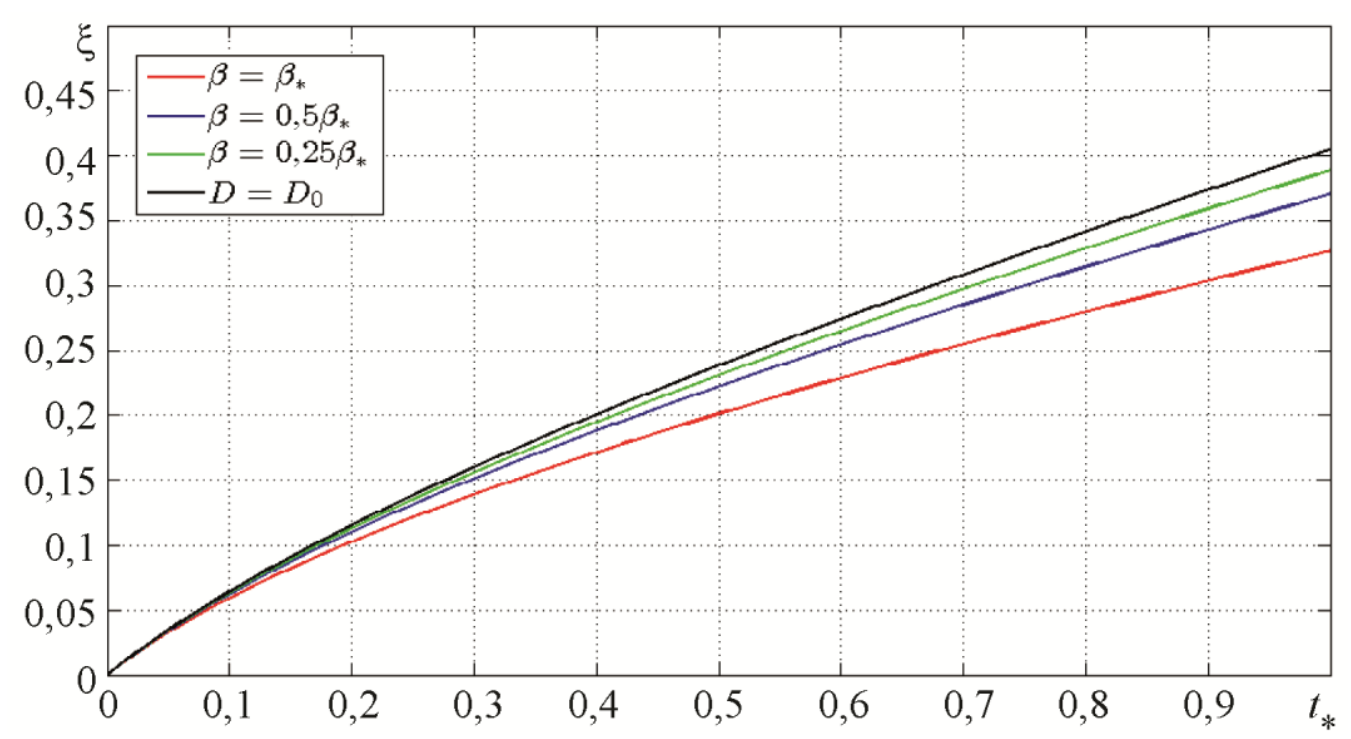

Рис. 6. Зависимость относительной толщины $\xi$ от времени $t_{*}$ для сферического тела при различных $\boldsymbol{\beta}, \boldsymbol{\gamma}=5 \boldsymbol{\gamma}_{*}$; заданные перемещения $\overline{u_{0}}=-0,01$.

Поскольку параметр $\boldsymbol{\beta}$ может варьироваться, рассмотрим, как его изменение будет влиять на кинетику фронта химической реакции. Относительное положение фронта хими- 
ческой реакции в разные моменты времени при различных значениях параметра приведено на рис. 6. Наибольшее расхождение с кинетикой фронта при постоянном коэффициенте диффузии наблюдается при $\boldsymbol{\beta}=\boldsymbol{\beta}_{*}$. Поскольку значение $\boldsymbol{\beta}_{*}$ было оценкой сверху, очевидно, что при уменьшении параметра разница между подходами будет становиться меньше и также можно пренебречь зависимостью коэффициента диффузии от напряжений.

\section{2. Кинетика фронта реакции в осесимметричном теле}

Кинетика фронта химической реакции в осесимметричном теле при различных внешних нагрузках была подробно рассмотрена в [25], здесь мы будем рассматривать только влияние выбора моделей диффузии.

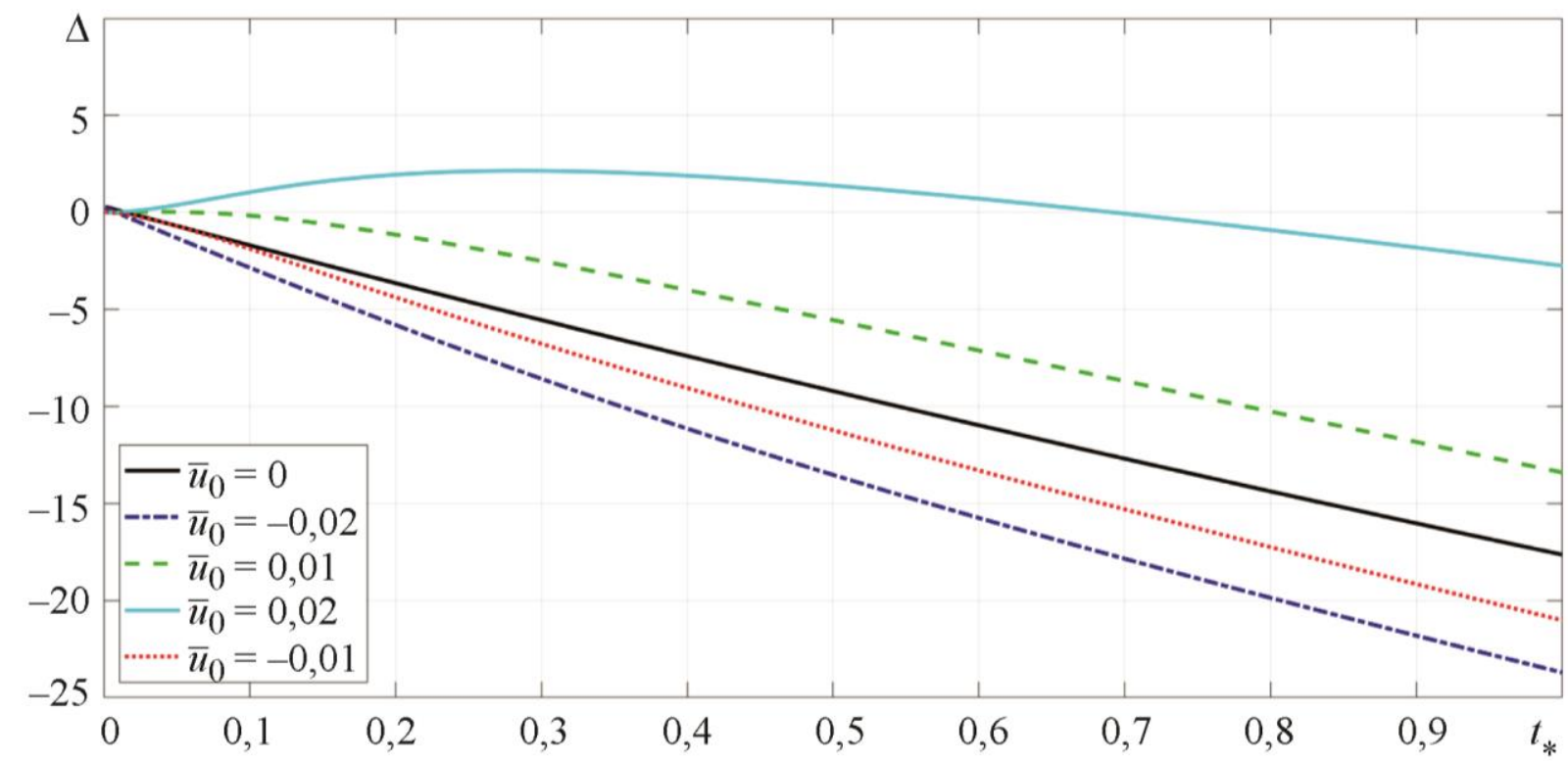

Рис. 7. Зависимость относительной разницы $\Delta$ от времени $t_{*}$ для осесимметричного тела,

$$
\gamma=5 \gamma_{*} \text {; заданные перемещения }
$$

Начнем рассмотрение с феноменологического коэффициента диффузии (12) при заданных перемещениях. На рис. 7 приведена относительная разница между толщинами слоя превращенного материала при учете зависимости коэффициента диффузии от напряжений и постоянным коэффициентом диффузии. За исключением случая значительных растягивающих деформаций, учет зависимости коэффициента диффузии от напряжений замедляет химическую реакцию. Разница между подходами становится тем значительнее, чем больше деформации на сжатие. Таким образом, и в этой краевой задаче при заданных перемещениях разница между подходами велика и не может быть проигнорирована.

Такое же поведение сохраняется и для заданных на границе усилий: учет зависимости коэффициента диффузии от напряжений, как и в случае заданных перемещений, замедляет рост превращенного слоя. При сжимающих напряжениях разница между подходами максимальна, при растягивающих - минимальна, однако, хотя разница между толщинами слоя превращенного материала в случае заданных напряжений меньше, чем в случае заданных перемещений, она все же недостаточно мала, чтобы ею можно было пренебречь.

Рассмотрим теперь влияние коэффициента диффузии (15), зависящего от деформаций скелета превращенного материала, на распространение фронта химической реакции. Как видно из кинетики роста относительной толщины превращенного слоя при заданных на границе перемещениях (рис. 8), использование коэффициента диффузии в виде (15) ускоряет 
продвижение фронта химической реакции в случае растягивающей деформации, а в случае сжимающей деформации - замедляет. При этом при одинаковых значениях сжимающей и растягивающей деформации, разница между кинетикой фронта с коэффициентом диффузии, заданным по формуле (15), и кинетикой с постоянным коэффициентом диффузии, больше в случае сжимающих деформаций.

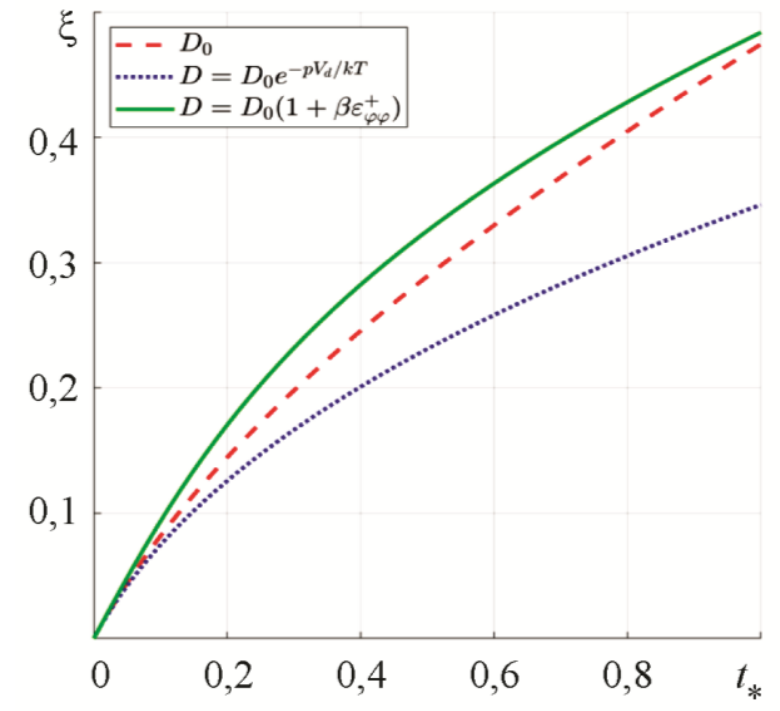

$a$

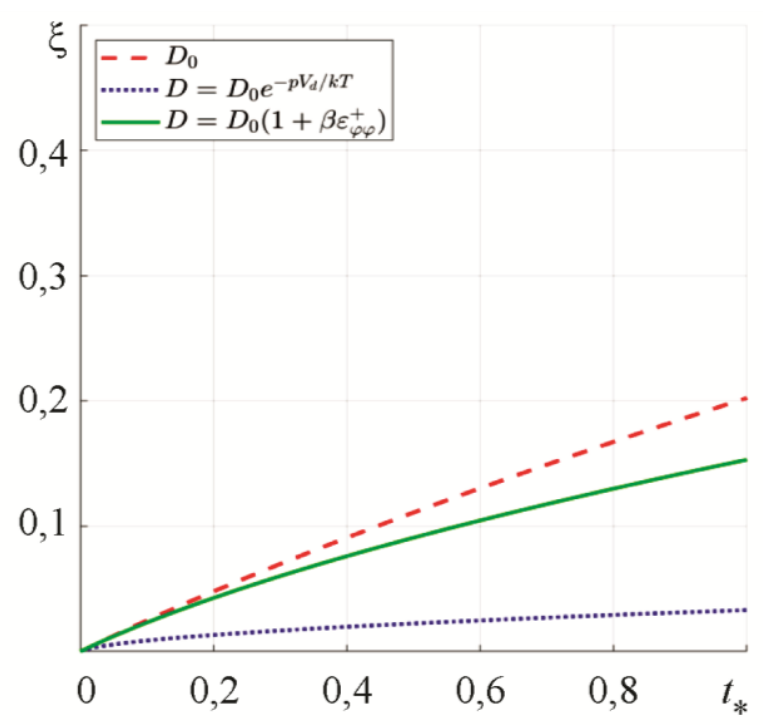

$\sigma$

Рис. 8. Зависимость относительной толщины $\boldsymbol{\xi}$ от времени $t_{*}$ для осесимметричного тела,

$$
\boldsymbol{\beta}=\boldsymbol{\beta}_{*}, \boldsymbol{\gamma}=5 \boldsymbol{\gamma}_{*} \text {; заданные перемещения на растяжение (a) и сжатие (б) }
$$

В обоих случаях различие между ростом толщины превращенного слоя с предложенным коэффициентом диффузии (15) и постоянным коэффициентом меньше, чем между ростом толщины слоя с коэффициентом, введенным эмпирически и постоянным коэффициентом диффузии, что более нагляднопредставлено для разных зависимостей коэффициента диффузии от напряжений (рис. 9). При этом, независимо от знака перемещений, разница между кинетикой толщины превращенного слоя с коэффициентом диффузии (15) и кинетикой с постоянным коэффициентом диффузии не превышает 5 \%, поэтому зависимостью коэффициента диффузии от напряжений, связанных с деформацией твердого тела можно пренебречь при проведении расчетов, не требующих высокой точности.

Результаты для заданных напряжений аналогичны результатам, которые были получены при заданных деформациях. Феноменологический коэффициент диффузии (13) замедляет распространение фронта химической реакции и оказывает более существенное влияние, чем тензорный коэффициент диффузии (15). Как и в предыдущих случаях, при выборе модели диффузии в виде (15) зависимостью диффузий от напряжений можно пренебречь. 


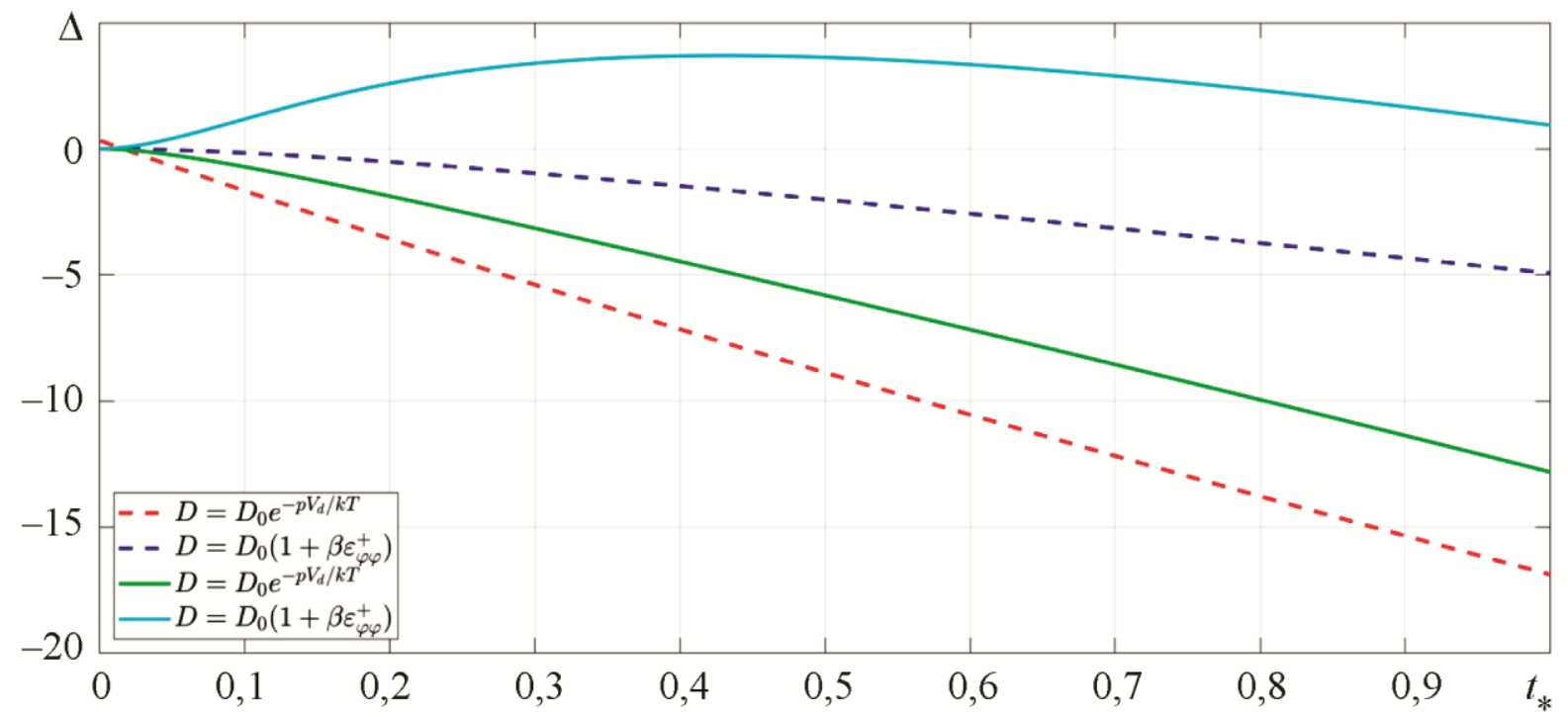

Рис. 9. Зависимость относительной разницы $\Delta$ от времени $t_{*}$ для осесимметричного тела, заданные деформации на растяжение (сплошные линии) и на сжатие (пунктирные линии)

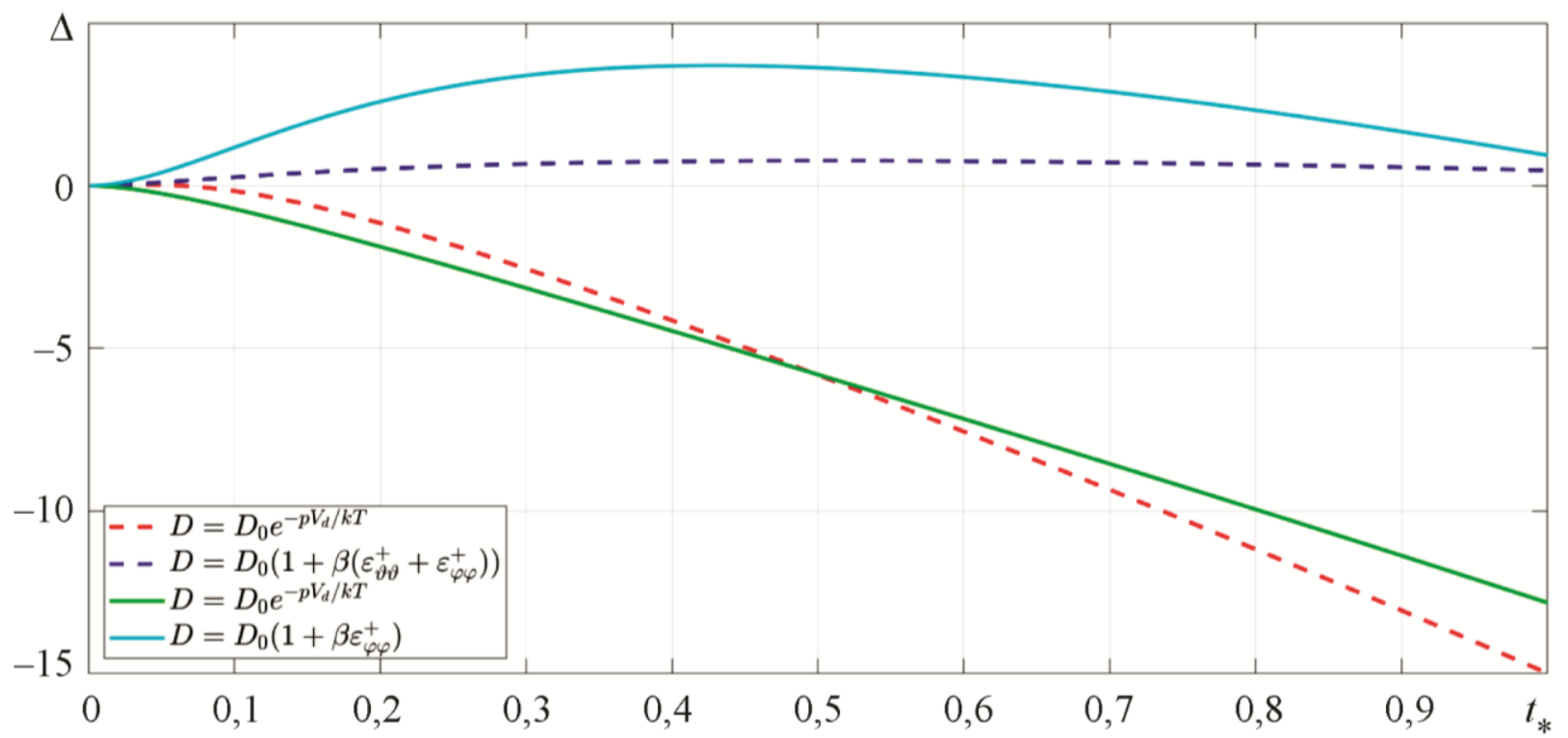

Рис. 10. Зависимость относительной разницы $\Delta$ от времени $t_{*}$ для одинаковых значений соответствующего энергетического параметра; заданные деформации на растяжение в осесимметричном теле (сплошныле линии) и в сфере (пунктирные линии)

Сравнение результатов для двух краевых задач при заданных растягивающих перемещениях приведено на рис. 10. Различие во влиянии учета зависимости диффузии от напряжений в центрально-симметричной и осесимметричной задачах связано, прежде всего, с гипотезой о плоско-деформированном состоянии, принятой в осесимметричной задаче.

\section{4. Выводы}

В этой работе мы исследовали влияние учета различных видов зависимости коэффициента диффузии от механических напряжений на скорость распространения фронта химической реакции. Помимо классической зависимости коэффициента диффузии от давления, была предложена модель диффузии, зависящая от деформаций твердого тела в плоскости, перпендикулярной направлению диффузионного потока, что привело к модели тензодиффу- 
зии. Проведено исследование кинетики продвижения фронта химической реакции в зависимости от приложенных внешних нагрузок для двух краевых задач: тела со сферической симметрией и осесимметричного тела в плоско-деформированном состоянии. Было проведено сравнение результатов для всех трех коэффициентов диффузии: постоянного, заданного эмпирически и предложенного в этой работе.

В случае заданных на границе деформаций учет зависимости коэффициента диффузии от давления оказывает значительное влияние на кинетику распространения фронта химической реакции. Это происходит вследствие дополнительной положительной деформации химического превращения, возникающей в результате химической реакции. Поскольку при заданных (фиксированных) перемещениях на границе тело не может расшириться ни в одном из направлений, то внутри превращенного материала возникают значительные сжимающие внутренние напряжения, которые приводят к значительному уменьшению коэффициента диффузии (13). В целом плоско-деформированное состояние в цилиндре оказывает большее влияние на диффузию газа к фронту химической реакции, чем это наблюдается в центрально-симметричном теле (рис. 10).

В случае заданных напряжений на границах тела, как для цилиндра, так и для шара, разница между кинетикой роста превращенного слоя при постоянном и эмпирическом коэффициентами диффузии значительно меньше, чем при жестком нагружении. Если работы требуют качественной оценки или неточной количественной, в этом случае можно пренебречь зависимостью коэффициента диффузии от напряжений и облегчить дальнейшие вычисления, так как использование постоянного коэффициента диффузии уменьшает количество необходимых параметров модели и, соответственно, количество экспериментов и расчетов, необходимых для нахождения этих параметров. Тем не менее, учет зависимости коэффициента диффузии от напряжений в виде (13) оказывает значительное влияние на кинетику реакции и не может быть проигнорирован при точных расчетах.

Предложенная модель тензодиффузии в независимости от типа граничных условий оказывает меньшее влияние на скорость распространения фронта химической реакции, чем эмпирически заданная зависимость коэффициента диффузии от напряжений. Это происходит потому, что значительный вклад в давление, от которого зависит эмпирический коэффициент диффузии, вносит радиальная компонента тензора напряжений. В то же время, поскольку в рассматриваемых задачах радиальная компонента напряжений действует в направлении диффузионного потока, она не оказывает существенного влияния на скорость распространения диффузионного потока, определяемого деформациями материала в плоскости, перпендикулярной $\mathbf{e}_{r}$. Таким образом, при выборе модели тензодиффузии скорость распространения фронта реакции определяется, прежде всего, скоростью химической реакции, а не диффузионным процессом и, как следствие, в этом случае влиянием механических напряжений на диффузионную кинетику можно пренебречь.

\section{Благодарность}

Исследование выполнено при поддержке гранта Российского научного фонда (проект № 18-19-00160).

\section{Литература}

1. Two-dimensional silicon oxidation experiments and theory / Dah-Bin Kao, J. P. McVittie, W. D. Nix, K. C. Saraswat // 1985 International Electron Devices Meeting. - IEEE, 1985. - P. 388-391.

2. Two-dimensional thermal oxidation of silicon-I. Experiments / Dah-Bin Kao, J. P. McVittie, W. D. Nix, K. C. Saraswat // IEEE Transactions on Electron Devices. - 1987. Vol. 34, iss. 5. - P. 1008-1017. - DOI: 10.1109/T-ED.1987.23037.

3. Sutardja P., Oldham W. G. Modeling of stress effects in silicon oxidation // IEEE Transactions on Electron Devices. - 1989. - Vol. 36, no. 11. - P. 2415-2421. - DOI: 10.1109/16.43661. 
4. Thermodynamics, diffusion and the Kirkendall effect in solids / Aloke Paul, Tomi Laurila, Vesa Vuorinen, Sergiy V. Divinski. - Springer, 2014.

5. Cui Z., Gao F., Qu J. Interface-reaction controlled diffusion in binary solids with applications to lithiation of silicon in lithium-ion batteries // Journal of the Mechanics and Physics of Solids. - 2013. - Vol. 61, no. 2. - P. 293-310. - DOI: 10.1016/j.jmps.2012.11.001.

6. Knyazeva A. G. Model of medium with diffusion and internal surfaces and some applied problems // Mater. Phys. Mech. - 2004. - Vol. 7, no. 1. - P. 29-36.

7. Knyazeva A. G. Cross effects in solid media with diffusion //Journal of applied mechanics and technical physics. - 2003. - Vol. 44, no. 3. - P. 373-384. - DOI: 10.1023/A:1023485224031.

8. Role of drawing-induced residual stresses and strains in the hydrogen embrittlement susceptibility of prestressing steels / J. Toribio, V. Kharin, M. Lorenzo, D. Vergara // Corrosion Science. 2011. - Vol. 53, no. 10. - P. 3346-3355. - DOI: 10.1016/j.corsci.2011.06.012.

9. Cui Z., Gao F., Qu J. A finite deformation stress-dependent chemical potential and its applications to lithium ion batteries // Journal of the Mechanics and Physics of Solids. - 2012. - Vol. 60, no. 7. - P. 1280-1295. - DOI: 10.1016/j.jmps.2012.03.008.

10. Freidin A. B. Chemical affinity tensor and stress-assist chemical reactions front propagation in solids // ASME 2013 International Mechanical Engineering Congress and Exposition. - American Society of Mechanical Engineers, 2013. - P. V009T10A102-V009T10A102.

11. Freidin A. B. On the chemical affinity tensor for chemical reactions in deformable materials // Mechanics of Solids. - 2015. - Vol. 50, no. 3. - P. 260-285. - DOI: 10.3103/S0025654415030048.

12. Chemical affinity tensor and chemical reaction front propagation: theory and FE-simulations / Alexander B. Freidin, Igor K. Korolev, Sergey P. Aleshchenko, Elena N. Vilchevskaya // International Journal of Fracture. - 2016. - Vol. 202, no. 2. - P. 245-259. - DOI: 10.1007/s10704-016-0155-1.

13. Freidin A. B., Vilchevskaya E. N., Korolev I. K. Stress-assist chemical reactions front propagation in deformable solids // International Journal of Engineering Science. - 2014. - Vol. 83. P. 57-75. - DOI: 10.1016/j.ijengsci.2014.03.008.

14. Chemical reactions in spherically symmetric problems of mechanochemistry / A. Freidin, N. Morozov, S. Petrenko, E. Vilchevskaya // Acta Mechanica. - 2016. - Vol. 227, no. 1, pp. 43-56. DOI: $10.1007 / \mathrm{s} 00707-015-1423-2$.

15. Glansdorff P., Prigogine I. Thermodynamic theory of structure, stability and fluctuations // American Journal of Physics. - 1973. - Vol. 41, no. 1. - P. 147-148.

16. Thermodynamic Methods in the Theory of Heterogeneus Systems / ed. by M. J. Grinfel'd. Longman Scientific and Technical, 1991.

17. Two-dimensional thermal oxidation of silicon. II. Modeling stress effects in wet oxides / Dah-Bin Kao, J. P. McVittie, W. D. Nix, K. C. Saraswat // IEEE transactions on electron devices. 1988. - Vol. 35, no. 1. - P. 25-37. - DOI: 10.1109/16.2412.

18. Loeffel K., Anand L. A chemo-thermo-mechanically coupled theory for elastic-viscoplastic deformation, diffusion, and volumetric swelling due to a chemical reaction // International Journal of Plasticity. - 2011. - Vol. 27, no. 9. - P. 1409-1431. - DOI: 10.1016/j.ijplas.2011.04.001.

19. Yen J. Y., Hwu J. G. Stress effect on the kinetics of silicon thermal oxidation // Journal of Applied Physics. - 2001. - Vol. 89, no. 5. - P. 3027-3032. - DOI: 10.1063/1.1342801.

20. Indeitsev D., Mochalova Y. Mechanics of multi-component media with exchange of mass and non-classical supplies // Dynamics of Mechanical Systems with Variable Mass. - Springer, Vienna, 2014. - P. 165-194.

21. Deal B. E., Grove A. S. General relationship for the thermal oxidation of silicon // Journal of Applied Physics. - 1965. - Vol. 36, no. 12. - P. 3770-3778.

22. Delph T. J. Intrinsic strain in SiO 2 thin films // Journal of Applied Physics. - 1998. Vol. 83, no. 2. - P. 786-792. - DOI: 10.1063/1.366759.

23. Lin M. T. Stress effects and oxidant diffusion in the planar oxidation : thesis and dissertation. - Lehigh University, 1999. 
24. Vilchevskaya E. N., Freidin A. B., Morozov N. F. Kinetics of the chemical reaction front in centrally symmetric problems of mechanochemistry // Doklady Physics. - 2015. - Vol. 60, iss. 4. P. 175-179. - DOI: 10.1134/S1028335815040072.

25. Vilchevskaya E. N., Freidin A. B. On kinetics of chemical reaction fronts in elastic solids // Surface effects in solid mechanics. - Springer, Berlin, Heidelberg, 2013. - P. 181-194. 